\title{
Immunohistochemical localisation of thrombospondin in human megakaryocytes and platelets
}

\author{
KATHRYN M MCLAREN \\ From the Department of Pathology, University Medical School, Teviot Place, Edinburgh EH8 9AG, Scotland
}

SUMMARY Thrombospondin (TSP) is a glycoprotein released from platelets after thrombininduced aggregation. Endothelial culture studies suggest that it or a similar protein may also be produced by endothelial cells. The origin of the glycoprotein is therefore of some importance. This report concerns the immunohistochemical localisation of TSP within megakaryocytes and platelets. TSP would appear to be a primary platelet protein of megakaryocyte origin, rather than a substance taken up by the platelet in the circulation. The relation of this molecule to the endothelial glycoprotein remains to be established, but our preliminary immunohistochemical studies suggest that the molecule is also located in vascular endothelium.

Thrombospondin (TSP) is a glycoprotein thought to be contained in the $\alpha$ granule fraction of human platelets and released after thrombin-induced aggregation. ${ }^{12}$ It has been suggested that it is very similar to a high molecular weight glycoprotein secreted by bovine aortic ${ }^{3}$ and human umbilical vein ${ }^{4}$ endothelial cells in culture.

The source of the protein is of importance. If it is synthesised only in endothelial cells then one must postulate uptake of the protein into the platelet in plasma, as may occur with serotonin. ${ }^{5}$ If, however, the protein is synthesised in megakaryocytes it may be designated a primary platelet secretory protein. The molecule may be synthesised by both cells independently or perhaps taken up by endothelium from plasma. We have obtained a specific antiserum to the human platelet-derived TSP and have used this for TSP localisation. This paper reports the first demonstration by immunofluorescence technique of TSP within megakaryocytes in human bone marrow, and within platelets in both marrow and plasma.

\section{Material and methods}

Thrombospondin was isolated from platelets as previously described ${ }^{6}$ and the glycoprotein purified. Antiserum to the isolated antigen was raised in the rabbit, as for radioimmunoassay. 6 Smears of human bone marrow aspirates were used for megakaryo-

Accepted for publication 2 September 1982 cyte study. They included cases of chronic myeloid leukaemia and of histologically normal marrow, obtained during staging procedure for nonHodgkin's lymphoma. No conditions of known platelet abnormality were used. Platelet-rich plasma (PRP) prepared so as to prevent platelet aggregation $^{7}$ was smeared on glass slides. Localisation involved the use of an indirect immunofluorescence method. $^{8}$ The TSP antiserum was used at a dilution of $1 / 20$. As a control, normal rabbit serum was used in place of primary antiserum.

\section{Results}

All marrow specimens showed intense fluorescent staining of megakaryocyte cytoplasm (Fig. 1). The staining could be resolved as a granular cytoplasmic deposit presumably reflecting platelet formation within the demarcation membrane system (Fig. 2). This was most obvious in those megakaryocytes exhibiting proplatelet extensions, wherein the fluorescence adopted a discrete granular staining pattern. The PRP showed a similar intense fluorescence over platelet cytoplasm. Lack of resolution prevented subcellular localisation vithin the cytoplasm: thus specific $\alpha$-granule localisation could not be confirmed. However, samples of platelets studied after induction of the release reaction by thrombin addition to PRP failed to stain in this immunofluorescence assay.

Marrow and non-aggregated platelet-rich plasma 


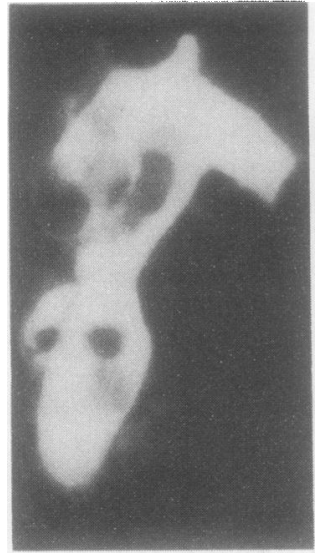

Fig. 1

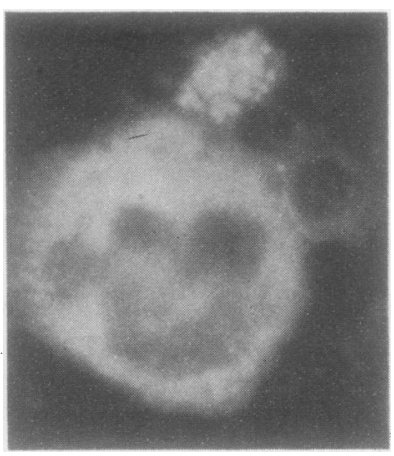

Fig. 2

Fig. 1 Megakaryocyte in bone marrow exhibits intense, granular cytoplasmic immunoftuorescence. Indirect immunofuorescence. TSP antiserum $\times 1400$

Fig. 2 The discrete granular immunoftuorescence at the periphery of the megakaryocyte represents platelet staining in a proplatelet extension. Indirect immunofuorescence. TSP antiserum $\times 1400$

treated with normal rabbit serum similarly failed to stain. In marrow sections no cell apart from megakaryocyte and platelet showed immunofluorescent staining.

\section{Discussion}

This is the first demonstration of TSP within parent megakaryocyte as well as in platelets by an immunohistochemical method. The glycoprotein was first described as a platelet product released after thrombin-induced aggregation. ${ }^{12}$ Subcellular localisation must await electronmicroscopic investigation but our findings of abolition of staining after induction of the release reaction, as well as TSP isolation studies ${ }^{2910}$ suggest that the product is contained within $\alpha$ granules. Its presence in megakaryocytes is particularly important in view of reports suggesting that the molecule is very similar to one secreted by bovine ${ }^{3}$ and human ${ }^{4}$ endothelial cells in culture. It has been proposed that the platelet source may represent uptake after release by endothelium into plasma. ${ }^{11} \mathrm{~A}$ similar mechanism exists for serotonin, ${ }^{5}$ but subsequent storage is in the dense bodies; sequestration into $\alpha$ granules would be unique. Since other constituents of the $\alpha$ granule are of megakaryocyte origin and probably represent synthetic products formed in the Golgi apparatus and subsequently packaged as secretory granules, secondary packaging in such organelles after uptake

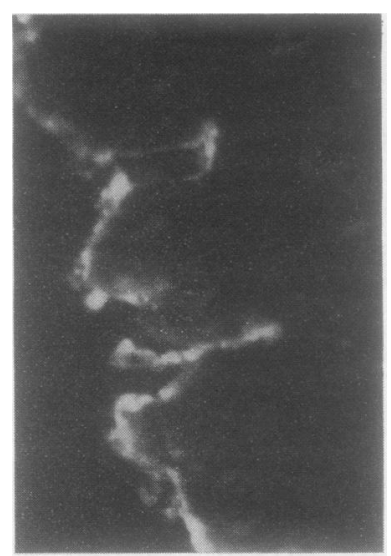

Fig. 3 Endothelium of human renal artery exhibits intense fluorescence. Indirect immunofuorescence. TSP antiserum $\times 800$

by platelets would seem unlikely. Thus the molecule is, like other granule products such as $\beta$ thromboglobulin and platelet factor $4,,^{1213}$ present in the megakaryocyte cytoplasm and in the platelet in both marrow and plasma sites.

The role of TSP remains unresolved but platelet aggregation studies suggest that the glycoprotein may be bound to the platelet surface after thrombin stimulation. ${ }^{14}$ It may function as a lectin, ${ }^{15}$ binding to the surface fibrinogen of platelets during thrombin-mediated aggregation. ${ }^{16}$ The high molecular weight of TSP and its migration position on gel electrophoresis suggest similarity to $\alpha-2$ macroglobulin and fibronectin, but from immunological evidence it is clearly a distinct molecule. ${ }^{11}$ Studies of the endothelial glycoprotein suggest that such high molecular weight products are associated with the surface of the cell ${ }^{17}$ but are principally found on its abluminal aspect. Since it seems that TSP is of primary platelet origin, it may, like factor VIII antigen and fibronectin, be produced by both megakaryocyte/platelet and endothelium. This concept would be consistent with a role for TSP in platelet endothelial co-operation, likened to the permissive action of factor VIII in mediating platelet adhesion, ${ }^{18}$ its presence in platelets and endothelium being a prerequisite of platelet/vessel wall interaction.

It is, however, possible that endothelial siting represents secondary uptake, a concept which is also consistent with a role for TSP in platelet vessel wall interaction, analogous to the endothelial binding by platelet proteins such as PF4. ${ }^{1920}$ Such uptake may have functional importance. Thus PF4 may compete with low density lipoproteins for endothelial binding sites, ${ }^{21}$ and modulate subsequent uptake. 
Reports suggest that TSP may be produced by both bovine ${ }^{3}$ and human ${ }^{4}$ endothelial cells in culture. It is therefore important that the nature of the endothelial protein be determined. Studies were carried out in order to establish whether the antiserum, in a comparable technique to that used to demonstrate megakaryocyte and platelet localisation, reveals endothelial staining. Preliminary results suggest that endothelia of main and interlobular renal arteries show positive fluorescence (Fig. 3). The staining appears to show cytoplasmic localisation with an additional subendothelial band closely related to the abluminal aspect of the cells. The exact parenchymal distribution of TSP-for example, details of glomerular localisation, and the relation of the apparent cytoplasmic to the extracellular staining remain to be determined.

Thrombospondin therefore represents another platelet protein synthesised within the megakaryocyte and released from the platelet after thrombininduced aggregation. Questions remain not only about its in vivo function but also about its relation to a similar molecule associated with vascular endothelium. The molecule and antisera directed against it may provide additional tools in the investigation of platelet function, thrombus formation and the complex interaction of platelets and the vessel wall.

Acknowledgements are due to Dr J Dawes, MRC Radioimmunoassay Unit, Forrest Road, Edinburgh who was responsible for the production of the thrombospondin antibody. The work herein described was supported by a grant from the Wellcome Trust.

\section{References}

' Baenziger NS, Brodie GN, Majerus PW. Isolation and properties of a thrombin-sensitive protein of human platelets. $J$ Biol Chem 1972;247:2723-31.

${ }^{2}$ Hagen I, Olsen T, Solum NO. Studies on subcellular fractions of human platelets by lactoperoxidase-iodination technique. Biochim Biophys Acta 1976;455:214-25.

${ }^{3}$ Sage H, Crouch E, Bornstein P. Collagen synthesis by bovine aortic endothelial cells in culture. Biochemistry 1979; 18:5433-42.

${ }^{4}$ Mosher DF, Doyle MJ, Jaffe EA. Synthesis and secretion of thrombospondin by cultured human endothelial cells. J Cell Biol 1982;93:343-8.
${ }^{5}$ Stacy RS. Uptake of 5-hydroxytryptamine by platelets. $\mathrm{Br} \mathrm{J}$ Pharmacol 1961;16:284-95.

- Dawes J, Clemetson KJ, Gogstad GO, et al. A radioimmunoassay for thrombospondin, used in a comparative study of thrombospondin, $\beta$-thromboglobulin and platelet factor 4 in healthy volunteers. Thromb Res (submitted for publication).

' Ludlam CA, Cash JD. Studies on the liberation of $\beta$-thromboglobulin from human platelets in vitro. Br J Haematol 1976;33:239-47.

8 Piovella F, Ascari E, Sitar GM, et al. Immunofluorescent detection of factor VIII-related antigen in human platelets and megakaryocytes. Haemostasis 1974;3:288-95.

9 Gerrard JM, Phillips DR, Rao GHR, et al. Biochemical studies of two patients with the gray platelet syndrome-selective deficiency of platelet alpha granules. $J$ Clin Invest 1980;66:102-9.

${ }^{10}$ Gogstad GO, Hagen I, Korsmo R, Solum NO. Characterisation of the proteins of isolated human platelet $\alpha$-granules. Evidence for a separate $\alpha$-granule-pool of the glycoproteins $\mathrm{II}_{\mathrm{b}}$ and III $_{\mathrm{a}}$. Biochim Biophys Acta 1981;670:150-62.

${ }^{11} \mathrm{McPherson} J$, Sage $\mathbf{H}$, Bornstein P. Isolation and characterisation of a glycoprotein secreted by aortic endothelial cells in culture. $J$ Biol Chem 1981;21:11330-5.

12 Ryo R, Proffitt RT, Poger HE, O'Bear R, Deuel TF. Platelet factor 4 antigen in megakaryocytes. Thromb Res 1980;17:645-52.

${ }^{13}$ McLaren KM, Pepper DS. The immunological localisation of $\beta$-thromboglobulin and platelet factor 4 in human megakaryocytes and platelets. J Clin Pathol 1982;35:1227-31.

${ }^{14}$ Phillips DR, Jennings LK, Prasanna HR. $\mathrm{Ca}^{2+}$-mediated association of glycoprotein $G$ (thrombin-sensitive protein, thrombospondin) with human platelets. J Biol Chem 1980;255:11629-32.

is Jaffé EA, Leung LLK, Nachman RL, Levin RI, Mosher DF. Thrombospondin is the endogenous lectin of human platelets. Nature 1982;295:246-8.

${ }^{16}$ Gartner TK, Gerrard JM, White JG, Williams DC. Fibrinogen is the receptor for the endogenous lectin of human platelets. Nature 1981;289:688-90.

${ }^{17}$ Birdwell CR, Gospodarowicz D, Nicolson GL. Identification, localisation and role of fibronectin in cultured bovine endothelial cells. Proc Natl Acad Sci USA 1978;75:3273-7.

18 Jaffé EA. Endothelial cells and the biology of factor VIII. $N$ Engl J Med 1977;296:377-83.

${ }^{19}$ Busch C, Dawes J, Pepper DS, Wasteson A. Binding of platelet factor 4 to cultured human endothelial cells. Thromb Haemost 1979;42:43, 89 (Abstr).

${ }^{20}$ Dawes J, Pumphrey CW, McLaren KM, Prowse CV, Pepper DS. The in vitro release of human platelet factor 4 by heparin. Thromb Res 1982;27:65-76.

${ }^{21}$ Brown MS, Deuel TF, Basu SK, Goldstein JL. Inhibition of the binding of low-density lipoprotein to its cell surface receptor in human fibroblasts by positively charged proteins. J Supramol Struct 1978;8:223-34.

Requests for reprints to: Dr Kathryn M McLaren, Department of Pathology, University Medical School, Teviot Place, Edinburgh EH8 9AG, Scotland. 